\title{
Human Tyrosine Hydroxylase Natural Allelic Variation: Influence on Autonomic Function and Hypertension
}

\author{
Fangwen Rao $\cdot$ Kuixing Zhang $\cdot$ Lian Zhang $\cdot$ Brinda K. Rana $\cdot$ \\ Jennifer Wessel $\cdot$ Maple M. Fung $\cdot$ Juan L. Rodriguez-Flores • \\ Laurent Taupenot $\cdot$ Michael G. Ziegler $\cdot$ Daniel T. O'Connor
}

Received: 17 May 2010/ Accepted: 4 June 2010/Published online: 23 June 2010

(C) The Author(s) 2010. This article is published with open access at Springerlink.com

\begin{abstract}
The catecholamine biosynthetic pathway consists of several enzymatic steps in series, beginning with the amino acids phenylalanine and tyrosine, and eventuating in the catecholamines norepinephrine (noradrenaline) and epinephrine (adrenaline). Since the enzyme tyrosine hydroxylase (TH; tyrosine 3-mono-oxygenase; EC 1.14.16.2; chromosome $11 \mathrm{p} 15.5$ ) is generally considered to be rate-limiting in this pathway, probed as to whether common genetic variation at the $T H$ gene occurred, and whether such variants contributed to inter-individual alterations in autonomic function, either biochemical or physiological. We began with sequencing a tetranucleotide (TCAT) repeat in the first intron, and found that the two most common versions, $\left(\right.$ TCAT) 6 and $(\text { TCAT })_{10 i}$, predicted heritable autonomic traits in twin pairs. We then conducted systematic polymorphism discovery across the $\sim 8 \mathrm{kbp}$ locus, and discovered numerous variants, principally non-coding. The proximal promoter block contained four common variants, and its haplotypes and SNPs (especially C-824T,
\end{abstract}

Fangwen Rao, Kuixing Zhang, and Lian Zhang contributed equally.

A commentary to this article can be found at doi:10.1007/s10571-010-9607-8.

F. Rao $\cdot$ K. Zhang $\cdot$ L. Zhang $\cdot$ B. K. Rana $\cdot$ J. Wessel $\cdot$

M. M. Fung · J. L. Rodriguez-Flores · L. Taupenot ·

M. G. Ziegler · D. T. O'Connor $(\bowtie)$

Departments of Medicine, Pharmacology, and Psychiatry, University of California at San Diego, Skaggs (SSPPS) room 4256, 9500 Gilman Drive, La Jolla, CA 92093-0838, USA e-mail: doconnor@ucsd.edu

F. Rao $\cdot$ K. Zhang $\cdot$ L. Zhang $\cdot$ B. K. Rana $\cdot$ J. Wessel $\cdot$ M. M. Fung · J. L. Rodriguez-Flores · L. Taupenot ·

M. G. Ziegler · D. T. O'Connor

VA San Diego Healthcare System, Skaggs (SSPPS) room 4256, 9500 Gilman Drive, La Jolla, CA 92093-0838, USA rs10770141) predicted catecholamine secretion, environmental stress-induced BP increments, and hypertension. Finally, we found that two of the common promoter variants, C-824T (rs10770141) and A-581G (rs10770140), were functional in that they differentially affected transcriptional activity of the isolated promoter, disrupted recognition motifs for specific transcription factor binding, altered the promoter responses to the co-transfected (exogenous) factors, and bound the endogenous factors in the chromatin fraction of the nucleus. We concluded that common variation in the proximal $T H$ promoter is functional, giving rise to changes in autonomic function and consequently cardiovascular risk.

Keywords Catecholamine $\cdot$ Stress $\cdot$ Chromaffin · Catecholamine

\author{
Abbreviations \\ PC12 Rat pheochromocytoma cell line \\ TH Tyrosine hydroxylase (tyrosine 3-mono- \\ oxygenase;EC 1.14.16.2) \\ TCAT Tetranucleotide repeat in $T H$ intron-A
}

\section{Introduction}

Tyrosine hydroxylase ( $T H$; tyrosine 3-mono-oxygenase; EC 1.14.16.2; chromosome $11 \mathrm{p} 15.5$ ), which catalyzes the formation of DOPA from dopamine, is generally considered to be the rate-limiting enzyme in the catecholamine biosynthetic pathway (Flatmark and Stevens 1999). The human $T H$ locus, on chromosome 11p15 (Chitbangonsyn et al. 2003), contains 13 exons/ 12 introns spanning approximately $8 \mathrm{kbp}$. Although a single-copy gene, $T H$ produces four different 
types of mRNA through alternative splicing of a single primary transcript (Grima et al. 1987), indicating one novel level of regulating catecholamines. Substantial loss of $T H$ enzymatic activity has profound consequences both in humans (Flatmark and Stevens 1999) and in mice with targeted ablation of the $T H$ locus (Zhou et al. 1995).

\section{TH Microsatellite Polymorphism and Hypertension}

Essential (idiopathic) hypertension develops over several decades of life. The human $T H$ locus bears several examples of common natural allelic variation, such as the tetranucleotide repeat [or microsatellite polymorphism, $(\mathrm{TCAT})_{n}$ ] in its first intron, which associate with essential hypertension (Sharma et al., 1998). The TH tetranucleotide repeat $(\mathrm{TCAT})_{n}$ occurs in 5-11 head-to-tail copies. Barbeau et al. (2003) found that (TCAT) 6 and (TCAT) ${ }_{10 \mathrm{i}}$ alleles seemed to be cardioprotective by association with attenuation of the hemodynamic response to stress with increasing age, while $(\mathrm{TCAT})_{7}$ seemed to be deleterious by their association with higher resting systolic blood pressure and greater hemodynamic response to stress with increasing body mass index. Sharma et al. (1998) found that the (TCAT) 10 ("E") allele was over-represented in hypertensive subjects, whereas the $(\mathrm{TCAT})_{9}$ ("D") allele frequency was increased in control (normotensive) subjects. Wei et al. (1997) found (TCAT) 9 to be associated with higher norepinephrine levels.

In order to probe the role of $T H$ polymorphism in stressinduced disease pathways, we used a classical human twin study design (Boomsma et al. 2002). Exploring the effects of particular (TCAT) $)_{n}$ alleles on "intermediate phenotypes," we stratified each individual on the basis of number of copies of the allele. We found that the two most common alleles, (TCAT) 6 and (TCAT) $)_{10 i}$, influenced a number of autonomic traits, both biochemical and physiological: $(\text { TCAT })_{6}$ copy number affected basal pulse interval and heart rate, and post-stress heart rate. (TCAT) $10 \mathrm{i}$ copy number affected basal pulse interval, plasma epinephrine, and renal norepinephrine excretion (Zhang et al. 2004). We documented the heritability of blood pressure, and found that (TCAT) ${ }_{6}$ allele frequencies differed among individuals stratified by genetic risk (family history) of hypertension: in particular, family history-positive individuals were less likely to bear the $(\mathrm{TCAT})_{6}$ allele. Since increasing $(\text { TCAT })_{6}$ allele copy number was associated with lower basal and stress-induced heart rates, the results obtained suggested a mechanism whereby (TCAT) 6 alleles may be protective against the future development of hypertension.

The (TCAT) $n$ motif can bind such transcription factors as $\mathrm{AP}^{10}$ or ZNF191 (Albanese et al. 2001) and may function as a transcriptional enhancer when tested in transfected/expressed promoter/reporter plasmids. However, in transfected $T H$ promoter/intron/reporter studies (Albanese et al. 2001), the (TCAT) ${ }_{n}$ repeat silences transcription in a copy number-dependent way; by contrast, in vivo we observed directionally opposite associations of common $(\mathrm{TCAT})_{n}$ alleles with autonomic function: $(\mathrm{TCAT})_{10 \mathrm{i}}$ with activation and (TCAT) 6 with diminution of sympathetic outflow (Zhang et al. 2004). Thus, transcriptional effects of the $(\mathrm{TCAT})_{n}$ could not mechanistically explain common variation in human autonomic function. Therefore, we carried out systematic polymorphism discovery at the locus.

\section{Discovery of Common $\mathrm{TH}$ Variants Governing Transcription, Human Autonomic Activity and Blood Pressure In Vivo}

In order to probe the possible underlying impact of TH variation on stress-induced disease pathways, we re-sequenced $\sim 1.2 \mathrm{kbp}$ of $5^{\prime}$ promoter as well as all 13 exons and adjacent intronic regions for rare and common variants in 80 ethnically diverse subjects as well as 422 twins. Plasma and urine catecholamines were measured by radioenzymatic assay (Kennedy and Ziegler 1990).

In the coding region, we found two common bi-allelic variants, only one of which was non-synonymous: Val81Met (at 37.4\%) which did not associate with the autonomic traits we studied. We also found 12 unusual coding region variants, but the non-synonymous variants had minor allele frequencies of only $0.3-0.6 \%$, not sufficient to account for the common population associations.

Thus, we turned to potential regulatory (non-coding) variants and found 10 SNPs in the proximal promoter region, four of which (C-824T, G-801C, A-581G, and G-494A) were common (minor allele frequencies $>10 \%$ ). Statistically, C-824T (rs10770141) and A-581G (rs10770140) seemed to influence both catecholamine secretion and the blood pressure response to environmental stress. A multivariable analysis further indicated that $\mathrm{C}-824 \mathrm{~T}$ became the most significant predictor of change in DBP during cold stress. The -824T allele is associated with increased catecholamine production, increased blood pressure increments in response to stress, and extreme blood pressure values in the population. It was tempting to speculate that the functional variation that we observed in $-824 \mathrm{~T}$ carriers could be the outcome of environmental selective pressures on alleles augmenting catecholaminergic function (Rao et al. 2007).

\section{Mechanistic Studies of Human TH Promoter Genetic Variation}

More recently, we performed additional studies to test whether such $T H$ promoter variants were by themselves 
functional (Zhang et al. 2010). First, common haplotypes (at least $1-5 \%$ frequency) of the $T H$ promoter were generated by site-directed mutagenesis, then verified by sequencing, and inserted into luciferase reporter vectors. After transfection into PC12 cells, such human TH promoter haplotypes showed substantial differences in luciferase reporter activity. By two-way ANOVA on luciferase activity, two of the four common variants (C-824T [rs10770141] and A-581G [rs10770140]) altered transcriptional activity. Comparison of these in cells results with the effects of the same 4 variants in vivo indicates that the same variants (C-824T and A-581G) that exert the greatest effect on $\mathrm{TH}$ transcription in cells also have the most pronounced effect on human catecholamine secretion in vivo. In addition, the $-824 \mathrm{~T}$ allele displayed an augmented response to typical chromaffin cell secretory stimuli, such as nicotine or PACAP. Under basal circumstances, greater activity of the $\mathrm{T}$ allele was observed, and when stimulated by nicotine, C-824T responded differentially, with an increased effect of the drug on the $\mathrm{T}$ allele; when stimulated by PACAP, the increased response of the $\mathrm{T}$ allele was even more apparent. The $-581 \mathrm{G}$ allele also responded differentially to PACAP. In the basal state, greater activity of the $\mathrm{G}$ allele was apparent; during nicotine stimulation, greater activity of the $\mathrm{G}$ allele was noted, as was the case during response to PACAP. We further probed the functional significance of C-824T and A-581G by co-transfection of sequence-predicted trans-activating factors in cells: MEF2, SRY, and FOXD1 differentially activated C-824T, whereas the G/C-rich binding factors SP1, AP2, and EGR1 differentially activated A-581G. At $\mathrm{C}-824 \mathrm{~T}$, the co-transfected human MEF2 increased $\mathrm{TH}$ promoter expression to $131.3 \%$ on the $\mathrm{T}$ allele, but had little effect on the $\mathrm{C}$ allele. The MEF2 dominant-negative mutant $\mathrm{mMEF} 2$ decreased $\mathrm{TH}$ promoter expression down to $71.3 \%$ on the $\mathrm{T}$ allele, but had little effect on the $\mathrm{C}$ allele. Thus, at $\mathrm{C}-824 \mathrm{~T}$, factor $\mathrm{MEF} 2$ acted in a directionally coordinate fashion (at $\mathrm{T}>\mathrm{C}$ ) to explain the in vivo trait associations, whereas at A-581G, factors SP1, AP2, and EGR1 displayed similar differential actions (at $\mathrm{G}>\mathrm{A}$ ). Chromatin immunoprecipitation (ChIP) confirmed the interaction of the endogenous transcription factors with these motifs in the nucleus (Zhang et al. 2010).

\section{Conclusions}

We conclude that inter-individual variability in catecholamine secretion is controlled in substantial part by genetic variation in the adrenergic pathway encoding cate cholamine synthesis, especially at the classical ratelimiting step, TH. TH promoter common polymorphisms C-824T and A-581G are not only statistical predictors of catecholamine secretory and stress BP response traits in vivo, but also causally responsible for alterations in transcriptional efficiency of the $T H$ gene. Our results thus document novel pathophysiological links between a key adrenergic locus, catecholamine metabolism, and blood pressure, and suggest new strategies to approach the mechanism, diagnosis, and treatment of autonomic dysfunction as well as systemic hypertension.

Acknowledgments This study was supported by grant from National Institutes of Health (HL58120, GCRC RR000827, Comprehensive Research Center of Excellence in Minority Health and Health Disparities [CRCHD], MD00020), and Department of Veterans Affairs. The authors declare no conflicts of interest.

Open Access This article is distributed under the terms of the Creative Commons Attribution Noncommercial License which permits any noncommercial use, distribution, and reproduction in any medium, provided the original author(s) and source are credited.

\section{References}

Albanese V, Biguet NF, Kiefer H, Bayard E, Mallet J, Meloni R (2001) Quantitative effects on gene silencing by allelic variation at a tetranucleotide microsatellite. Hum Mol Genet 10:1785-1792

Barbeau P, Litaker MS, Jackson RW, Treiber FA (2003) A tyrosine hydroxylase microsatellite and hemodynamic response to stress in a multi-ethnic sample of youth. Ethn Dis 13:186-192

Boomsma D, Busjahn A, Peltonen L (2002) Classical twin studies and beyond. Nat Rev Genet 3:872-882

Chitbangonsyn SW, Mahboubi P, Walker D, Rana BK, Diggle KL, Timberlake DS, Parmer RJ, O'Connor DT (2003) Physical mapping of autonomic/sympathetic candidate genetic loci for hypertension in the human genome: a somatic cell radiation hybrid library approach. J Hum Hypertens 17:319-324

Flatmark T, Stevens RC (1999) Structural insight into the aromatic amino acid hydroxylases and their disease-related mutant forms. Chem Rev 99:2137-2160

Grima B, Lamouroux A, Boni C, Julien JF, Javoy-Agid F, Mallet J (1987) A single human gene encoding multiple tyrosine hydroxylases with different predicted functional characteristics. Nature 326:707-711

Kennedy B, Ziegler MG (1990) A more sensitive and specific radioenzymatic assay for catecholamines. Life Sci 47:2143-2153

Rao F, Zhang L, Wessel J, Zhang K, Wen G, Kennedy BP, Rana BK, Das M, Rodriguez-Flores JL, Smith DW, Cadman PE, Salem RM, Mahata SK, Schork NJ, Taupenot L, Ziegler MG, O'Connor DT (2007) Tyrosine hydroxylase, the rate-limiting enzyme in catecholamine biosynthesis: discovery of common human genetic variants governing transcription, autonomic activity, and blood pressure in vivo. Circulation 116:993-1006

Sharma P, Hingorani A, Jia H, Ashby M, Hopper R, Clayton D, Brown MJ (1998) Positive association of tyrosine hydroxylase microsatellite marker to essential hypertension. Hypertension 32:676-682

Wei J, Ramchand CN, Hemmings GP (1997) Possible association of catecholamine turnover with the polymorphic $(\text { TCAT) })_{n}$ repeat in the first intron of the human tyrosine hydroxylase gene. Life Sci 61:1341-1347

Zhang L, Rao F, Wessel J, Kennedy BP, Rana BK, Taupenot L, Lillie EO, Cockburn M, Schork NJ, Ziegler MG, O’Connor DT (2004) 
Functional allelic heterogeneity and pleiotropy of a repeat polymorphism in tyrosine hydroxylase: prediction of catecholamines and response to stress in twins. Physiol Genomics 19:277-291

Zhang K, Zhang L, Rao F, Brar B, Rodriguez-Flores JL, Taupenot L, O'Connor DT (2010) Human tyrosine hydroxylase natural genetic variation: delineation of functional transcriptional control motifs disrupted in the proximal promoter. Circ Cardiovasc Genet 3:187-198

Zhou QY, Quaife CJ, Palmiter RD (1995) Targeted disruption of the tyrosine hydroxylase gene reveals that catecholamines are required for mouse fetal development. Nature 374:640-643 